\title{
Uso antropogénico, hábitat, abundancia y hábitos alimentarios del armadillo de nueve bandas (Dasypus novemcinctus) en el centro y sur del estado de Veracruz, México
}

\author{
Lorena López-de Buen, Felipe Zárate-Ledesma, Concepción Ahuja-Aguirre ${ }^{1}$, Citlalli \\ Vázquez-Morales, Apolo A. Carrasco-García y Felipe Montiel-Palacios
}

Facultad de Medicina Veterinaria y Zootecnia, Universidad Veracruzana, Circunvalación S/N Esq. Yáñez, Col. Unidad Veracruzana, C.P.91710, Veracruz, Veracruz, México. E-mail: lorelopez@uv.mx (LLB), felipezarate@uv.mx (FZL), cahuja@uv.mx (CAA), citlallivazquez@uv.mx (CVM), acarrasco@uv.mx (ACG),fmontiel@uv.mx (FMP)

${ }^{1}$ Autor para correspondencia

Resumen En las zonas rurales de México el armadillo de nueve bandas (Dasypus novemcinctus) es utilizado como alimento. El objetivo del estudio fue determinar el uso que los habitantes de ranchos ganaderos del estado de Veracruz, México, hacen de la especie, su hábitat, abundancia en la zona y hábitos alimentarios. En los años 2011 y 2016 se entrevistó a trabajadores de 33 ranchos de 10 municipios del centro y sur de Veracruz. En 2011 reportaron presencia del armadillo en 85\% de los ranchos y todos los municipios, con frecuencia de avistamiento semanal a anual. En todos los ranchos se aprovechaba el armadillo como alimento o ingreso económico. En cuatro municipios tenían vedas en la temporada reproductiva, y fuera de ésta cazaban solamente ejemplares adultos. Los entrevistados consideraron que había disminuido el número de armadillos en la zona. En 2016 reportaron presencia del armadillo en todos los ranchos, con frecuencia de avistamiento mensual y anual. Todos los entrevistados habían consumido armadillo, y todos los ejemplares habían sido cazados en la zona en temporada no reproductiva. En todos los ranchos predominó el estrato herbáceo. Se estimó abundancia de 19 y 24 armadillos $/ \mathrm{km}^{2}$ en el centro y sur, respectivamente. La dieta de los armadillos consistió principalmente de escarabajos, hormigas y mariposas. El armadillo de nueve bandas es utilizado como fuente de alimento en zonas rurales de Veracruz y debido a que es la presa que más cazan y consumen en la zona, muy posiblemente sus poblaciones han disminuido.

Palabras clave: abundancia, cobertura vegetal, consumo, Dasypodidae, densidad

Anthropogenic use, habitat, abundance, and feeding habits of the nine-banded armadillo (Dasypus novemcinctus) in central and southern Veracruz, Mexico

Abstract In rural areas in Mexico, the nine-banded armadillo (Dasypus novemcinctus) is used as food source. The aim of this study was to determine how this species is used by people living in cattle farms in the state of Veracruz, Mexico, as well as its habitat, abundance in the area, and feeding habits. In the years 2011 and 2016, workers from 33 cattle farms from 10 municipalities in central and southern Veracruz were interviewed. In 2011, they reported the presence of the armadillo in $85 \%$ of farms and all the municipalities, with weekly to annual sighting frequency. The armadillo was used as food or economic income in all farms. In four municipalities hunting is not practiced during the breeding season, and only adult individuals are caught during the hunting season. The farm workers considered that the number of armadillos in the area has decreased. In 2016, they reported the presence of armadillos on all farms, with weekly and annual sighting frequency. All workers interviewed had consumed armadillo meat, and all armadillos had been hunted during the non -breeding season. The herbaceous stratum predominated in all farms. An abundance of 19 and 24 armadillos $/ \mathrm{km}^{2}$ was estimated in the central and southern regions, respectively. The diet of the armadillos consisted mainly of beetles, ants, and butterflies. The nine-banded armadillo is a source of food and medicine in rural areas of Veracruz, but its populations may have declined because it is the most hunted and consumed prey in the area.

Keywords: abundance, consumption, Dasypodidae, density, plant cover 


\section{INTRODUCCIÓN}

El armadillo de nueve bandas (Dasypus novemcinctus Linnaeus, 1758) es un mamífero mediano encontrado en el continente americano, desde el sur de los Estados Unidos hasta el norte de Argentina (Gardner, 2008; Loughry \& McDonough, 2013). Es una de las dos especies de armadillos existentes en México (Ramírez-Pulido et al., 2005), en donde se distribuye en zonas cálidas y templadas, en pastizales, matorral xerófilo y diversos tipos de bosque, y en suelos arenosos o arcillosos en donde construye madrigueras subterráneas (Ceballos \& Galindo, 1984).

Esta especie se alimenta preferentemente de escarabajos y hormigas, aunque complementa su dieta con pequeños anfibios, reptiles, tubérculos, e incluso huevos de aves (McBee \& Baker, 1982; Redford, 1986; Tyler et al., 1996; Layne, 2003; Butler et al., 2004; Staller et al., 2005). Se aparea una vez al año, y se reportan temporadas reproductivas de junio a agosto en el hemisferio norte (Job et al., 1984; Layne, 2003; Mengak, 2005), y entre agosto y noviembre en el hemisferio sur (Neris et al., 2002; CONANP, 2014). El nacimiento de las crías ocurre en primavera, y éstas siempre son cuatrillizos homocigotos del mismo sexo (Job et al., 1984; Layne, 2003; Mengak, 2005). Sus funciones ecológicas incluyen el ser fuente de alimento para depredadores y carroñeros, controlador de insectos plaga, proveedor de nutrientes para las plantas, bioindicador del cambio climático al expandir su distribución geográfica hacia lugares que han registrado aumento de temperatura, además de que sus madrigueras sirven de refugio para otros animales silvestres (Moeller, 1990; Layne, 2003; Bowles, 2008).

En áreas rurales el armadillo de nueve bandas es utilizado como alimento o para elaborar medicinas y artesanías (Enríquez et al., 2006; Alves et al., 2007; Monroy-Vilchis et al., 2008; Ávila-Nájera et al., 2011). En ciertas regiones es considerado como plaga porque afecta cultivos agrícolas (Moeller, 1990; Long, 2003), consume huevos de tortugas y aves, y excava un número considerable de agujeros en la tierra al buscar comida o hacer su madriguera (Ober et al., 2014). Además, es reservorio de patógenos tanto para animales domésticos, p. ej. Sarcocystis neurona (Tanhauser et al., 2001), como para el hombre, p. ej. Mycobacterium leprae (Paige et al., 2002; Loughry et al., 2009), Coccidioides immitis (Eulálio et al., 2000), Paracoccidioides brasiliensis (Bagagli et al., 2003; Nishikaku et al., 2008), Trypanosoma cruzi (Paige et al., 2002; Yeo et al., 2005), Toxoplasma gondii (da Silva et al., 2006) y Leishmania naiffi (Naiff et al., 1991). Todo esto, aunado a la caza y pérdida de hábitat, ha reducido sus poblaciones en México y lo ponen en riesgo de extinción local (Ceballos \& Oliva, 2005; SEMARNAT, 2012).

En México no hay datos sobre el estado actual de las poblaciones de esta especie (SEMARNAT, 2012), y no está listada en la Norma Oficial Mexicana relativa a la protección de especies nativas de México de flora y fauna silvestre (NOM-059-SEMARNAT-2010) ni en la Convención sobre el Comercio Internacional de Especies Amenazadas de Fauna y Flora Silvestres (CITES, 2017). La Unión Internacional para la Conservación de la Naturaleza (UICN) la considera de Preocupación Menor (Loughry et al., 2014).

El objetivo del estudio fue determinar el uso que dan al armadillo de nueve bandas los habitantes de ranchos ganaderos de la zona centro y sur del estado de Veracruz, México, así como su hábitat, abundancia en la zona y hábitos alimentarios.

\section{MATERIALES Y MÉTOdOS}

\section{Área de estudio}

El estudio se realizó en los años 2011 y 2016. En 2011 se visitaron 20 ranchos ganaderos, 10 de ellos ubicados en cinco municipios de la zona centro (Cotaxtla, Jamapa, Medellín, Soledad de Doblado y Veracruz), y 10 más en cinco municipios de la zona sur (Aguadulce, Las Choapas, Coatzacoalcos, Minatitlán y San Andrés) del estado de Veracruz, México (FIG. 1; TABLA 1). En la zona centro, Jamapa y Soledad de Doblado contaban con la mayor superficie agrícola, Veracruz con la mayor superficie urbana, Cotaxtla y Medellín con la mayor superficie de pastizales, y Cotaxtla y Soledad de Doblado con la mayor superficie de vegetación natural. Por su parte, en la zona sur, San Andrés tenía la mayor superficie agrícola, Coatzacoalcos la mayor superficie urbana, Agua Dulce y Minatitlán la mayor superficie de pastizales, y Las Choapas la mayor superficie de vegetación natural (TABLA 2).

Los ranchos se seleccionaron con base en las facilidades proporcionadas por los administradores para realizar los recorridos y recabar la información. Cinco años después, y con la finalidad de determinar si el armadillo de nueve bandas seguía presente en la misma área de estudio y si se le seguía dando el mismo uso por parte de los pobladores, se entrevistaron nuevamente trabajadores de siete ranchos de Cotaxtla y seis de Jamapa, no habiendo sido posible visitar los municipios de la zona sur.

\section{Uso antropogénico del armadillo}

En 2011 se entrevistaron a 35 personas, incluyendo habitantes o trabajadores de los 20 ranchos visitados y cazadores de armadillos en la zona. Se realizó una entrevista estructurada dirigida a conocer la presencia del armadillo de nueve bandas en el lugar, la frecuencia de avistamiento y el aprovechamiento que realizaban del mismo. Cuando los entrevistados afirmaron haberlo visto en los ranchos, se les preguntó con qué frecuencia lo hacían, y si le daban algún uso. En el caso de los cazadores de armadillo, se les preguntó con qué frecuencia lo cazaban, la manera 
TABLA 1. Ubicación, altitud y clima en 10 municipios del centro y sur del estado de Veracruz, México. Datos tomados de INEGI (2016).

\begin{tabular}{|c|c|c|c|c|c|}
\hline Municipio & Coordenadas & $\begin{array}{l}\text { Altitud } \\
\text { (msnm) }\end{array}$ & $\begin{array}{l}\text { Temperatura } \\
\text { anual }\end{array}$ & $\begin{array}{c}\text { Precipitación } \\
\text { anual }\end{array}$ & Clima \\
\hline \multicolumn{6}{|l|}{ Zona centro } \\
\hline Cotaxtla & $18^{\circ} 50^{\prime} 11^{\prime \prime} \mathrm{N}, 94^{\circ} 38^{\prime} 11^{\prime \prime} \mathrm{W}$ & 30 & $26^{\circ} \mathrm{C}$ & $1.900 \mathrm{~mm}$ & cálido-seco \\
\hline Jamapa & $19^{\circ} 02^{\prime} 28^{\prime \prime} \mathrm{N}, 96^{\circ} 14^{\prime} 28^{\prime \prime} \mathrm{W}$ & 10 & $25^{\circ} \mathrm{C}$ & $1.108 \mathrm{~mm}$ & cálido-seco \\
\hline Medellín & $19^{\circ} 03^{\prime} 42^{\prime \prime} \mathrm{N}, 96^{\circ} 09^{\prime} 44^{\prime \prime} \mathrm{W}$ & 10 & $26^{\circ} \mathrm{C}$ & $1.350 \mathrm{~mm}$ & cálido-subhúmedo \\
\hline Soledad & $19^{\circ} 02^{\prime} 47^{\prime \prime} \mathrm{N}, 96^{\circ} 25^{\prime} 16^{\prime \prime} \mathrm{W}$ & 100 & $25^{\circ} \mathrm{C}$ & $1.100 \mathrm{~mm}$ & cálido-subhúmedo \\
\hline Veracruz & $19^{\circ} 11^{\prime} 25^{\prime \prime} \mathrm{N}, 96^{\circ} 09^{\prime} 12^{\prime \prime} \mathrm{W}$ & 10 & $25^{\circ} \mathrm{C}$ & $1.350 \mathrm{~mm}$ & cálido-subhúmedo \\
\hline \multicolumn{6}{|l|}{ Zona sur } \\
\hline Agua Dulce & $18^{\circ} 08^{\prime} 17^{\prime \prime} \mathrm{N}, 94^{\circ} 08^{\prime} 37^{\prime \prime} \mathrm{W}$ & 10 & $26^{\circ} \mathrm{C}$ & $2.700 \mathrm{~mm}$ & cálido-húmedo \\
\hline Las Choapas & $17^{\circ} 54^{\prime} 31^{\prime \prime} \mathrm{N}, 94^{\circ} 05^{\prime} 54^{\prime \prime} \mathrm{W}$ & 10 & $26^{\circ} \mathrm{C}$ & $2.900 \mathrm{~mm}$ & cálido-húmedo \\
\hline Coatzacoalcos & $18^{\circ} 08^{\prime} 09^{\prime \prime} \mathrm{N}, 94^{\circ} 27^{\prime} 48^{\prime \prime} \mathrm{W}$ & 10 & $26^{\circ} \mathrm{C}$ & $2.600 \mathrm{~mm}$ & cálido-húmedo \\
\hline San Andrés & $18^{\circ} 26^{\prime} 29^{\prime \prime} \mathrm{N}, 95^{\circ} 12^{\prime} 51^{\prime \prime} \mathrm{W}$ & 280 & $24{ }^{\circ} \mathrm{C}$ & $3.500 \mathrm{~mm}$ & cálido-húmedo \\
\hline Minatitlán & $18^{\circ} 01^{\prime} 02^{\prime \prime} \mathrm{N}, 94^{\circ} 33^{\prime} 38^{\prime \prime} \mathrm{W}$ & 10 & $26^{\circ} \mathrm{C}$ & $2.600 \mathrm{~mm}$ & cálido-húmedo \\
\hline
\end{tabular}

TABLA 2. Población humana, superficie total, agrícola, urbana, de pastizales y de vegetación natural, y ecosistemas predominantes en municipios de Veracruz, México. Datos tomados de INEGI (2016).

\begin{tabular}{|c|c|c|c|c|c|c|c|}
\hline Municipio & $\begin{array}{l}\text { Población } \\
\text { humana } \\
\text { (No.) }\end{array}$ & $\begin{array}{l}\text { Superficie } \\
\text { total (ST) } \\
\left(\mathrm{km}^{2}\right)\end{array}$ & $\begin{array}{l}\text { Superficie } \\
\text { agrícola km² }^{2} \\
(\% \text { de ST })\end{array}$ & $\begin{array}{l}\text { Superficie } \\
\text { urbana } \mathrm{km}^{2} \\
(\% \text { de ST) }\end{array}$ & $\begin{array}{l}\text { Superficie } \\
\text { de pastizales } \\
\mathrm{km}^{2}(\% \text { de } \\
\text { ST })\end{array}$ & $\begin{array}{l}\text { Superficie con } \\
\text { vegetación } \\
\text { secundaria, } \\
\text { selva o } \\
\text { bosque } \mathrm{km}^{2} \\
(\% \text { de } \mathrm{ST})\end{array}$ & $\begin{array}{c}\text { Ecosistemas } \\
\text { predominantes }\end{array}$ \\
\hline \multicolumn{8}{|l|}{ Zona centro } \\
\hline Cotaxtla & 19.710 & 537 & $202(37,7)$ & $0,3(0,1)$ & $294(54,8)$ & $39(7,3)$ & Selva baja caducifolia \\
\hline Jamapa & 10.376 & 132 & $80(61,0)$ & $0,9(0,7)$ & $46(35,2)$ & $4(3,0)$ & Selva baja caducifolia \\
\hline Medellín & 59.126 & 398 & $158(39,7)$ & $2,3(0,5)$ & $220(55,2)$ & $15(4,0)$ & $\begin{array}{l}\text { Selva baja caducifolia, } \\
\text { bosque perennifolio }\end{array}$ \\
\hline Soledad & 27.008 & 416 & $244(58,6)$ & $1,9(0,4)$ & $139(33,4)$ & $31(7,4)$ & $\begin{array}{l}\text { Bosque subtropical } \\
\text { perennifolio }\end{array}$ \\
\hline Veracruz & 552.156 & 247 & $70(28,2)$ & $35,6(14,3)$ & $113(45,9)$ & $8(3,2)$ & $\begin{array}{l}\text { Bosque alto o } \\
\text { mediano tropical } \\
\text { perennifolio }\end{array}$ \\
\hline \multicolumn{8}{|l|}{ Zona sur } \\
\hline Agua Dulce & 20.973 & 372 & $51(13,7)$ & $11,0(2,9)$ & $270(72,6)$ & $20(5,4)$ & $\begin{array}{l}\text { Selva alta perennifo- } \\
\text { lia con palmares }\end{array}$ \\
\hline Las Choapas & 77.426 & 3509 & $93(2,6)$ & $12,0(0,3)$ & $2056(58,5)$ & $1271(36,2)$ & $\begin{array}{l}\text { Selva baja perennifo- } \\
\text { lia y caducifolia }\end{array}$ \\
\hline Coatzacoalcos & 305.260 & 309 & $9(2,9)$ & $50,3(16,2)$ & $140(45,4)$ & $52(17,0)$ & $\begin{array}{l}\text { Selva alta peren- } \\
\text { nifolia con pal- } \\
\text { mares, manglares y } \\
\text { pastizales }\end{array}$ \\
\hline San Andrés & 157.364 & 957 & $410(42,8)$ & $7,6(0,8)$ & $398(41,6)$ & $104(10,9)$ & $\begin{array}{l}\text { Selva alta peren- } \\
\text { nifolia con dunas } \\
\text { costeras y vegetación } \\
\text { secundaria }\end{array}$ \\
\hline Minatitlán & 157.840 & 2.115 & $82(3,9)$ & $29,1(1,3)$ & $1449(68,5)$ & $293(13,8)$ & $\begin{array}{l}\text { Bosque alto con } \\
\text { bejuco y epífitas }\end{array}$ \\
\hline
\end{tabular}




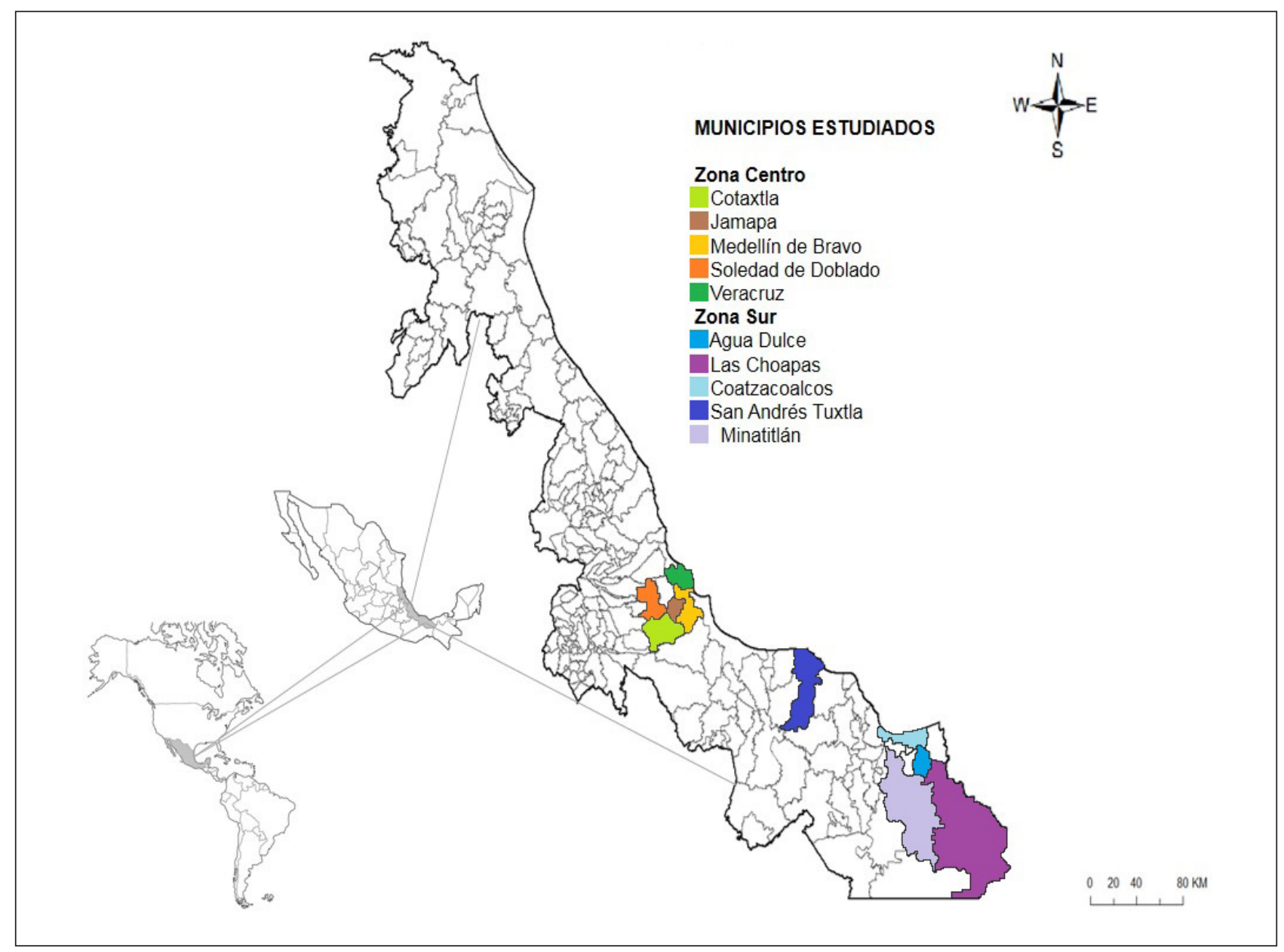

FIGURA 1. Mapa del estado de Veracruz, México, con los municipios incluidos en el estudio.

en que lo hacían y la cantidad de ejemplares capturados cada vez o en un periodo determinado. En 2016 se realizó la misma entrevista a 13 trabajadores, uno por cada uno de los 13 ranchos de Cotaxtla y Jamapa, para actualizar la información obtenida en el 2011 para esta zona.

\section{Cobertura vegetal}

Para conocer el hábitat del armadillo en el área de estudio, se determinó la cobertura vegetal de los ranchos visitados en 2011. De cada uno se obtuvieron las coordenadas (GPS Garmin GPSMap 60, Garmin Ltd., Kansas, USA) para ubicarlos satelitalmente y localizar las áreas con componente arbóreo en Google Earth. En éstas se establecieron por rancho seis parcelas de $100 \times 100 \mathrm{~m}$ cada una, procurando una separación mínima de 100 m entre parcela, según lo permitiera el paisaje, y tratando de cubrir la mayor proporción de área a donde se pudiera tener acceso caminando. Cada parcela fue georreferencia$\mathrm{da}$, delimitando los puntos cardinales en las esquinas. Posteriormente, por observación directa se determinó el porcentaje de cobertura vegetal a partir del análisis de componentes vegetativos, estimando la proporción de cada estrato (arbóreo, arbustivo y herbáceo), y asignando un valor a cada uno según la escala BraunBlanquet (Mueller-Dombois \& Ellenberg, 1974):
$1=5 \%$ de cobertura; $2=>5 \%$ a $25 \% ; 3=>25 \%$ a $50 \%$; $4=>50 \%$ a $75 \% ; y=>75 \%$ a $100 \%$.

\section{Estimación de abundancia}

En 2011 se estimó la abundancia del armadillo de nueve bandas en cada rancho mediante dos recorridos de transecto en zigzag ( $2.000 \mathrm{~m}$ largo x $50 \mathrm{~m}$ ancho) realizados en áreas arboladas, mismos que fueron georreferenciados. Los recorridos se hicieron en dos días consecutivos de 7:00 a 12:00 hs, por haber sido el horario en el que se nos permitió realizarlos. Cada uno duró cinco horas. Durante cada recorrido se trató de observar ejemplares, huellas (Aranda, 2000), o madrigueras con evidencia de actividad reciente (rastros de entrada y salida de animales). Se consideró como presencia de un individuo, cada avistamiento, como así también cuando se encontraron huellas de paso por el transecto o madrigueras activas (distancia mínima de $100 \mathrm{~m}$ entre una y otra). Esto se hizo ante la posibilidad de tener menos avistamientos durante el horario del recorrido, dados los hábitos nocturnos de la especie.

La abundancia del armadillo por zona se calculó de la siguiente manera:

Abundancia $=\Sigma$ individuos en los 20 transectos / $\mathrm{km}^{2}$ recorridos 
considerando que fueron dos transectos por rancho, de $0,1 \mathrm{~km}^{2}$ cada uno, y 10 ranchos por zona, lo que equivale a $2 \mathrm{~km}^{2}$ recorridos por zona.

\section{Hábitos alimentarios}

En 2011, y para conocer los hábitos alimentarios del armadillo en la zona, se analizaron 15 tractos digestivos de ejemplares donados por cazadores. Se llevó a cabo la identificación de los tipos de alimento consumido (ítems) encontrados siguiendo la metodología descrita por Maehr \& Brady (1986).

Los resultados del estudio se reportan de manera descriptiva.

\section{RESULTADOS Y DISCUSIÓN}

\section{Uso antropogénico del armadillo}

Los resultados de las encuestas aplicadas en 2011 indicaron la presencia del armadillo de nueve bandas en $90 \%$ de los ranchos en la zona centro (ausencia en uno de Veracruz) y en $80 \%$ de los ranchos en la zona sur (ausencia en uno de Coatzacoalcos y uno de Aguadulce). En los ranchos en donde se reportó la presencia del armadillo, la frecuencia de avistamiento varió desde un individuo a la semana hasta uno al año. A pesar de haberse reportado la presencia de esta especie en $85 \%$ de todos los ranchos, los entrevistados consideraron que era escasa, habiendo disminuido el número de ejemplares avistados y el de los ejemplares capturados, debido principalmente a la caza excesiva.

Además, en todos los ranchos se realizó aprovechamiento del armadillo como alimento o ingreso económico extra. En este último caso, era vendido para consumo o artesanía. En Cotaxtla, Medellín, Minatitlán y Veracruz los cazadores dijeron tener vedas en la temporada reproductiva, y fuera de ésta atrapar sólo ejemplares adultos, así como capturar al menos un animal por semana con perros entrenados. En este estudio, el armadillo de nueve bandas fue considerado por los cazadores como la presa que más cazan y más consumen. Esto es similar a lo que ocurre en otras regiones de México, como la selva Lacandona (Guerra \& Naranjo, 2003), la zona cafetalera del centro de Veracruz (Tlapaya \& Gallina, 2010), Oaxaca (Lira-Torres \& Briones-Salas, 2011) y San Luis Potosí (Ávila-Nájera et al., 2011), en donde esta especie es de las presas de mayor demanda y de la que se captura mayor número de ejemplares. En cuanto al método predominante de caza, el uso de perros también fue reportado en la zona cafetalera del centro de Veracruz (Tlapaya \& Gallina, 2010). Dado que este método no permite seleccionar a las presas por edad, tamaño ni sexo, los perros pueden atrapar crías o hembras gestantes, así como dejar malheridos a algunos ejemplares que mueren sin ser aprovechados (Tlapaya \& Gallina, 2010). Por lo tanto, este método de caza predominante en la zona podría considerarse como uno de los más perjudiciales para la supervivencia del armadillo.

Según los resultados de las encuestas aplicadas en 2016 en ranchos de Cotaxtla y Jamapa, todos los entrevistados habían visto armadillos, similar a lo que reportaron en 2011. La frecuencia de avistamiento fue mensual $(31 \%)$ y anual $(69 \%)$, menor a la mencionada en algunos ranchos en 2011, de un ejemplar a la semana. De los entrevistados, el 92\% cazaba armadillos, y de éstos, el $42 \%$ cazaba uno al año, $42 \%$ dos al año y $16 \%$ tres ejemplares al año. En cuanto al uso que le dieron a los armadillos capturados, todos los entrevistados indicaron haberlo consumido como alimento, el 85\% dijo haberlo usado también como medicina y el 15\% para realizar artesanías. Estos resultados fueron similares a los obtenidos en 2011.

Todos los entrevistados dijeron que los armadillos que consumen son cazados en la zona y que los capturan sólo en la temporada no reproductiva, similar a lo indicado en 2011. Según ellos, la temporada reproductiva ocurre en febrero $(8 \%)$, abril $(38 \%)$, mayo $(8 \%)$, julio (38\%), agosto $(23 \%)$ y septiembre $(8 \%)$, mientras que en la literatura se ha reportado que el apareamiento ocurre entre junio y noviembre (Job et al., 1984; Neris et al., 2002; Layne, 2003; Mengak, 2005; CONANP, 2014). Así, es posible que los entrevistados que piensan que la temporada reproductiva ocurre entre febrero y mayo capturen ejemplares, incluyendo hembras gestantes, el resto del año, cuando ocurre en realidad la temporada reproductiva. Esto podría contribuir a la disminución de las poblaciones en la zona en un mediano o largo plazo. Todos los entrevistados coincidieron en calificar a la especie como escasa en la zona, similar a lo que reportaron en 2011.

\section{Cobertura vegetal}

En los diversos ranchos de la zona centro de Veracruz predominó el estrato herbáceo (>75 a 100\%), seguido por el arbóreo ( $>5$ a $25 \%$ ), principalmente en los perímetros de las parcelas, y finalmente por el arbustivo (5\%), disperso en pequeños parches. En la zona sur también predominó el estrato herbáceo (>50 a 75\%), seguido por los estratos arbustivo y arbóreo ( $>5$ a $25 \%$ ). Estos resultados coinciden con los reportes que indican que en México, Veracruz es uno de los estados con mayor transformación de sus ecosistemas naturales, principalmente por la conversión en potreros, cultivos y zonas urbanas (Williams-Linera et al., 2002). Esto explica la predominancia del estrato herbáceo en los ranchos visitados, pues dado que el arbustivo es considerado como maleza, generalmente es eliminado.

\section{Estimación de abundancia}

En cada municipio se recorrieron $0,4 \mathrm{~km}$, totalizando $4 \mathrm{~km}$ en toda el área de estudio. Se contaron las madrigueras de armadillo con huellas recientes de actividad y los rastros o avistamientos de individuos. La presencia de ejemplares se confirmó en 


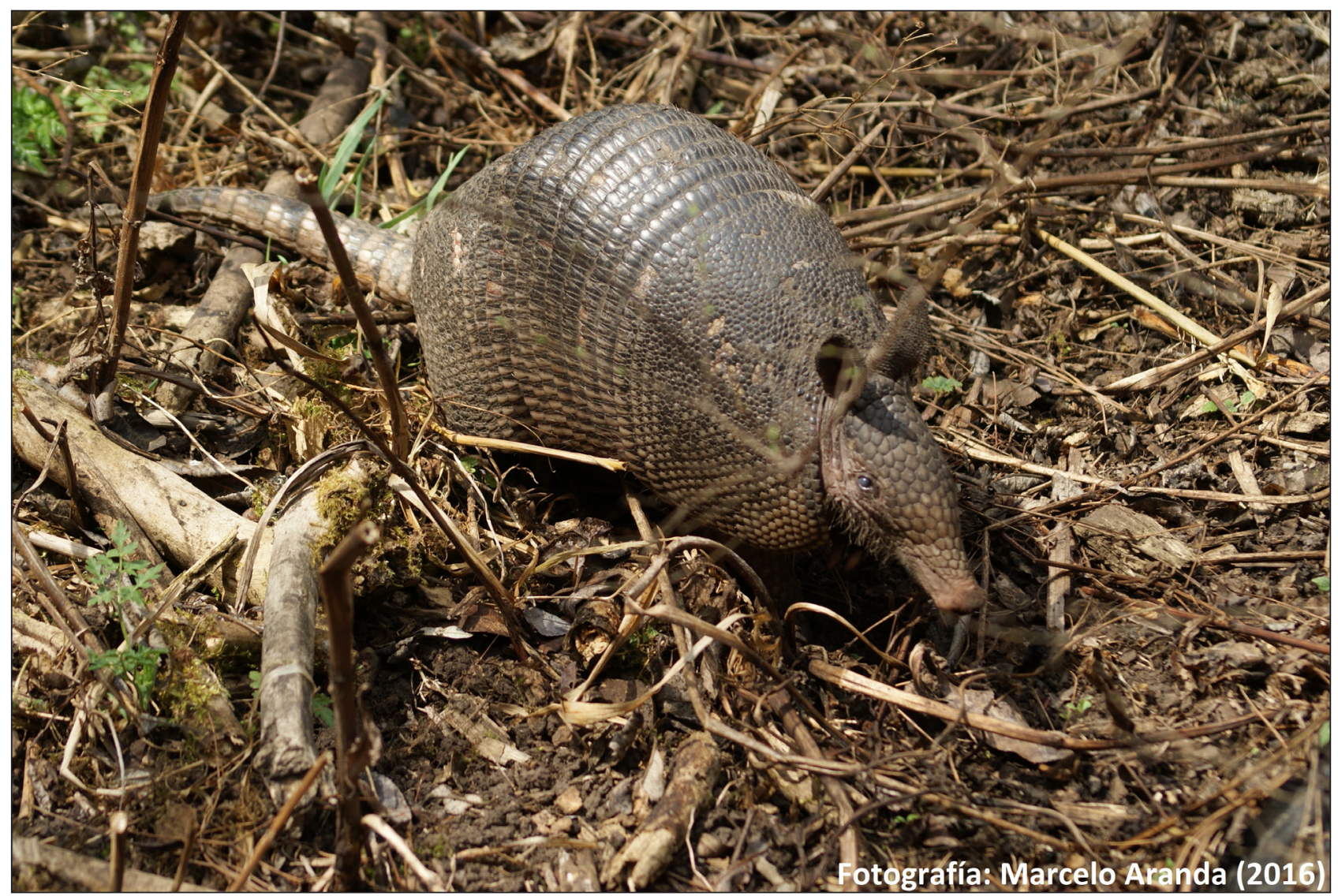

FIGURA 2. Ejemplar de armadillo de nueve bandas (Dasypus novemcinctus) observado en el municipio de San Andrés, en el estado de Veracruz, México.

todos los municipios. En la zona centro, se registraron siete madrigueras activas en Cotaxtla y Medellín pero no hubo avistamiento de ejemplares, mientras que en Jamapa y Soledad de Doblado se observaron un ejemplar y dos madrigueras activas por sitio. En la zona sur, en Minatitlán se observaron nueve madrigueras activas y 12 rastros, pero sin avistamiento de ejemplares; por la distancia entre ellas (en promedio 875 $\mathrm{m})$, se consideró que siete madrigueras pertenecían a distintos animales. En San Andrés se observaron dos armadillos adultos (FIG. 2) y cuatro madrigueras activas. Con base en estos datos, en la zona centro se estimó una abundancia de 19 armadillos $/ \mathrm{km}^{2}$ y en la zona sur 24 armadillos $/ \mathrm{km}^{2}$. En los municipios de la zona centro predominó el estrato herbáceo, mientras que en la zona sur la proporción de estrato arbóreo y arbustivo fue mayor que en la zona centro, aunque también predominó el estrato herbáceo. Esta mayor proporción de árboles y arbustos podría favorecer la presencia del armadillo en la zona sur.

En otros países se han estimado densidades de población de la especie muy variadas, desde $9,6 / \mathrm{km}^{2}$ en parches de bosque atlántico en Brasil en áreas con gran presión de caza, a 23,6/ $\mathrm{km}^{2}$ en un área protegida (Cullen et al., 2001). En Perú, en campos en donde se practica la tala y quema, la densidad estimada fue de $21,9 / \mathrm{km}^{2}$ (Naughton-Treves et al., 2003). Estas densidades son similares a las estimadas en este estudio.
Es posible que el aprovechamiento antropogénico también influya en la densidad de las poblaciones, pues en municipios como Aguadulce y Jamapa, donde la cobertura vegetal puede considerarse adecuada para $D$. novemcinctus, la presión de caza por los pobladores es fuerte, y podría contribuir a la disminución de sus poblaciones. En los municipios visitados hubo diferencias en la conformación natural del hábitat, en el tamaño de la población humana y en su grado de urbanización. Esto puede explicar la menor densidad de armadillos en Veracruz y Coatzacoalcos, municipios con mayor extensión de áreas urbanas (TABLA 2).

\section{Hábitos alimentarios}

$\mathrm{Al}$ analizar los 15 tractos digestivos de los armadillos donados por cazadores se encontraron 235 ítems, detectándose el consumo de insectos, principalmente de los órdenes Coleoptera (escarabajos; 78\%, 183/235), Hymenoptera (hormigas; 13\%, 31/235) y Lepidoptera (mariposas [restos de alas]; 6\%, 14/235), además de material vegetal $(2 \%, 5 / 235)$ y otros invertebrados $(1 \%, 3 / 235)$. Este consumo predominante de escarabajos, hormigas y mariposas concuerda con lo reportado por otros autores (Redford, 1986; Tyler et al., 1996; Anacleto, 2007) de que 90\% de la dieta de los armadillos se basa en insectos y otros artrópodos, e indica que en estas zonas el armadillo de nueve bandas mantiene los hábitos alimenticios descritos para la especie. 
La caza indiscriminada, junto con la deforestación y transformación del hábitat, son probablemente los factores que están afectando de manera más importante a las poblaciones de armadillo de nueve bandas en Veracruz, como ha sido señalado en décadas anteriores para otras especies de mamíferos en otras regiones (Robinson \& Redford, 1994). Generalmente, las especies más cazadas son las más comunes en el área y las que tienen altas tasas reproductivas (Bodmer, 1995; Bennett \& Robinson, 2000; Tlapaya \& Gallina, 2010), similar a lo observado con el armadillo de nueve bandas en Veracruz. El manejo adecuado de las diversas especies de animales silvestres puede llevar a su aprovechamiento sustentable (Robinson \& Bodmer, 1999; Robinson \& Bennett, 2000), como ha sido el caso del pecarí de collar (Tayassu tajacu) y del venado colorado o temazate (Mazama americana) en bosques tropicales de Perú, en donde ambas especies eran cazadas a tasas de cosecha anual menores que las tasas de cosecha sustentable (Alvard et al., 1997) sin que se produjera disminución de sus poblaciones. Esta misma estrategia de caza podría aplicarse para lograr el aprovechamiento sustentable del armadillo de nueve bandas en Veracruz.

\section{AGRADECIMIENTOS}

Al Consejo Nacional de Ciencia y Tecnología (CONACYT) de México, por la beca otorgada a uno de los autores para realizar estudios de maestría. A los propietarios de los ranchos por permitirnos el acceso a los mismos. A las personas que participaron respondiendo las encuestas.

\section{REFERENCIAS}

Alvard, M. S., J. G. Robinson, K. H. Redford \& H. Kaplan. 1997. The sustainability of subsistence hunting in the Neotropics. Conservation Biology 11: 977-982. http://dx.doi. org/10.1046/j.1523-1739.1997.96047.x

Alves, R. R. N., I. L. Rosa \& G. G. Santana. 2007. The role of animal-derived remedies as complementary medicine in Brazil. BioScience 57: 949-955. https: / / doi.org/10.1641 / B571107

Anacleto, T. C. S. 2007. Food habits of four armadillo species in the Cerrado area, Mato Grosso, Brazil. Zoological Studies 46: 529-537.

Aranda, M. 2000. Huellas y otros rastros de los mamíferos grandes y medianos de México. Instituto de Ecología, A. C., México. 212 pp.

Ávila-Nájera, D. M., O. C. Rosas-Rosas, L. A. Tarango-Arámbula, J. F. Martínez-Montoya \& E. Santoyo-Brito. 2011. Conocimiento, uso y valor cultural de seis presas del jaguar (Panthera onca) y su relación con éste, en San Nicolás de los Montes, San Luis Potosí, México. Revista Mexicana de Biodiversidad 82: 1020-1028.
Bagagli, E., M. Franco, S. M. G. Bosco, F. HebelerBarbosa, L. A. Trinca \& M. R. Montenegro. 2003. High frequency of Paracoccidioides brasiliensis infection in armadillos (Dasypus novemcinctus): an ecological study. Medical Mycology 41: 217223. https: / / doi.org/10.1080/136937803100015 97368

Bennett, E. L. \& J. G. Robinson. 2000. Hunting of wildlife in tropical forests. Implication for biodiversity and forest peoples. Environment Department working papers; Paper No. 76, Biodiversity Series. The World Bank Environment Department, Washington D.C. 42 pp. Disponible en <http:// documents.worldbank.org/curated / en / 101611468780290485 / Hunting-of-wildlife-in-tropical-forestsimplications-for-biodiversity-and-forestpeoples>

Bodmer, R. E. 1995. Managing Amazonian wildlife: biological correlates of game choice by detribalized hunters. Ecological Applications 5: 872877. http: / / dx.doi.org/10.2307/ 2269338

Bowles, E. 2008. The potential impacts of climate change on coastal North Carolina. A report by the Faculty of the University of North Carolina, Wilmington. November 28, 2008. Wilmington, North Carolina. 104 pp.

Butler, J. A., C. Broadhurst, M. Green \& Z. Mullin. 2004. Nesting, nest predation and hatchling emergence of the Carolina Diamondback Terrapin Malaclemys terrapin centrata in Northeastern Florida. American Midland Naturalist 152: 145-155. https: / / doi.org/10.167 4/0003-0031(2004)152[0145:NNPAHE]2.0.CO;2

Ceballos, G. \& C. Galindo. 1984. Mamíferos silvestres de la cuenca de México. Edit. Limusa, México. 299 pp.

Ceballos, G. \& G. Oliva (Eds.) 2005. Los mamíferos silvestres de México. Col. Sección de obras de ciencia y tecnología. FCE, CONABIO. 986 pp.

CITES. 2017. Convention on International Trade in Endangered Species of Wild Fauna and Flora. $<$ http://www.cites.org $>$. Consultada 2 de agosto de 2017.

CONANP - Comisión Nacional de Áreas Naturales Protegidas. México. 2014. <http://iztapopo. conanp.gob.mx/documentos/fichas_de_especies/Dasypus_novemcinctus.pdf $>$ Consultada 2 de octubre de 2017.

Cullen, L. Jr., R. E. Bodmer \& C. ValladaresPadua. 2001. Ecological consequences of hunting in Atlantic Forest patches, São Paulo, Brazil. Oryx 35: 137-144. http://dx.doi. org/10.1046/j.1365-3008.2001.00163.x

da Silva, A. V., S. M. G. Bosco, H. Langoni \& E. Bagagli. 2006. Study of toxoplasma infection 
in Brazilian wild mammals: serological evidence in Dasypus novemcinctus Linnaeus, 1758 and Euphractus sexcinctus Wagler, 1830. Veterinary Parasitology 135: 81-83. https:// doi.org/10.1016/j.vetpar.2005.08.013

Enríquez, V. P., R. Mariaca M., O. G. Retana G. \& E. J. Naranjo P. 2006. Uso medicinal de la fauna silvestre en Los Altos de Chiapas, México. Interciencia 31: 491-499.

Eulálio, K. D., R. L. Macedo, M. A. S. Cavalcanti, L. M. S. Martins, M. S. Lazéra \& B. Wanke. 2000. Coccidioides immitis isolated from armadillos (Dasypus novemcinctus) in the State of Piauí, northeast Brazil. Mycopathologia 149: 57-61. https: / / doi.org/10.1023 / A:1007273019647

Gardner, A. L. (ed.). 2008. Mammals of South America. Volume 1: marsupials, xenarthrans, shrews, and bats. University of Chicago Press, Chicago and London. 669 pp. https://doi. org / 10.7208/chicago/9780226282428.001.0001

Guerra, M. M. \& E. J. Naranjo. 2003. Cacería de subsistencia en dos localidades de la selva Lacandona, Chiapas, México. Pp. 339-344 in: Manejo de fauna silvestre en Amazonia y Latinoamérica (O. R. Polanco, ed.). Selección de trabajos V Congreso Internacional. CITES, Fundación Natura, Bogotá, Colombia.

INEGI - Instituto Nacional de Estadística y Geografía. 2016. Anuario estadístico y geográfico de los Estados Unidos Mexicanos 2016, México. 962 pp.

Job, C. K., R. M. Sanchez, W. F. Kirchheimer \& R. C. Hastings. 1984. Attempts to breed the ninebanded armadillo (Dasypus novemcinctus) in captivity - a preliminary report. International Journal of Leprosy and Other Mycobacterial Diseases 52: 362-364.

Layne, J. N. 2003. Armadillo: Dasypus novemcinctus. Pp. 75-97 in: Wild mammals of North America: biology, management and conservation (G. A. Feldhamer, B. C. Thompson \& J. A. Chapman, eds.). $2^{\text {nd }}$ ed. The Johns Hopkins University Press, Baltimore and London.

Lira-Torres, I. \& M. Briones-Salas. 2011. Impacto de la ganadería extensiva y cacería de subsistencia sobre la abundancia relativa de mamíferos en la Selva Zoque, Oaxaca, México. Therya 2: 217-244. https:/ / doi.org/10.12933/ therya- $11-49$

Long, J. L. 2003. Introduced mammals of the world: their history, distribution and influence. CSIRO Publishing, Collingwood, Australia. 589 pp.

Loughry, W. J. \& C. M. McDonough. 2013. The nine-banded armadillo: a natural history.
University of Oklahoma Press, Norman, Oklahoma, USA. 323 pp.

Loughry, W. J., C. M. McDonough \& A. M. Abba. 2014. Dasypus novemcinctus. The IUCN Red List of Threatened Species 2014: e.T6290A47440785. http: / / dx.doi.org/10.2305/IUCN.UK.20141.RLTS.T6290A47440785.en. Consultada 2 de agosto de 2017.

Loughry, W. J., R. W. Truman, C. M. McDonough, M. K. Tilak, S. Garnier \& F. Delsuc. 2009. Is leprosy spreading among nine-banded armadillos in the Southeastern United States? Journal of Wildlife Diseases 45: 144-152. https: / doi. org / 10.7589/0090-3558-45.1.144

Maehr, D. S. \& J. R. Brady. 1986. Food habits of bobcats in Florida. Journal of Mammalogy 67: 133-138. https: / / doi.org/10.2307 / 1381009

McBee, K. \& R. J. Baker. 1982. Dasypus novemcinctus. Mammalian Species 162: 1-9. https: / / doi. org $/ 10.2307 / 3503864$

Mengak, M. T. 2005. Nine-banded armadillo (Dasypus novemcinctus). University of Georgia Warner School of Forest Resources Publication - Natural History Series No. 4: 1-6. Disponible en <http:/ / digitalcommons.unl.edu/ icwdmother / 42>

Moeller, W. 1990. Modern xenarthrans. Pp. 583-626 in: Grzimek's Encyclopedia of Mammals, Vol. 2, English Language Edition (S. Parker, ed.). McGraw-Hill, Inc., New York.

Monroy-Vilchis, O., L. Cabrera, P. Suárez, M. M. Zarco-González, C. Rodríguez-Soto \& V. Urios. 2008. Uso tradicional de vertebrados silvestres en la Sierra Nanchititla, México. Interciencia 33: 308-313.

Mueller-Dombois, D. \& H. Ellenberg. 1974. Aims and methods of vegetation ecology. John Wiley \& Sons, New York. 547 pp.

Naiff, R. D., R. A. Freitas, M. F. Naiff, J. R. Arias, T. V. Barrett, H. Momen \& G. Grimaldi Jr. 1991. Epidemiological and nosological aspects of Leishmania naiffi Lainson \& Shaw 1989. Memorias do Instituto Oswaldo Cruz 86: 317-321. http://dx.doi.org/10.1590/ S0074-02761991000300006

Naughton-Treves, L., J. L. Mena, A. Treves, N. Alvarez \& V. C. Radeloff. 2003. Wildlife survival beyond park boundaries: the impact of slash-and-burn agriculture and hunting on mammals in Tambopata, Peru. Conservation Biology 17: 1106-1117. https://doi. org/10.1046/j.1523-1739.2003.02045.x

Neris, N., F. Colmán, E. Ovelar, N. Sukigara \& N. Ishii. 2002. Guía de mamíferos medianos y grandes del Paraguay: distribución, 
tendencia poblacional y utilización. Secretaría del Ambiente (SEAM), Agencia de Cooperación Internacional del Japón (JICA), Asunción, Paraguay. 165 pp.

Nishikaku, A. S., M. T. S. Peraçoli, E. Bagagli, M. F. Sugizaki \& A. Sartori. 2008. Experimental infections with Paracoccidioides brasiliensis obtained from armadillos: comparison to clinical isolates. Brazilian Journal of Infectious Diseases 12: 57-62. https:// doi.org/10.1590/ S1413-86702008000100013

Ober, H. K., L. W. DeGroote \& R. F. Mizell III. 2014. Baiting the nine-banded armadillo. Document WEC317, Wildlife Ecology and Conservation Department. IFAS Extension, University of Florida. Original publication December 2011; reviewed October 2014. 3 pp. Disponible en <http: / / edis.ifas.ufl.edu/uw362>

Paige, C. F., D. T. Scholl \& R. W. Truman. 2002. Prevalence and incidence of density of Mycobacterium leprae and Trypanosoma cruzi infections within a population of wild ninebanded armadillos. American Journal of Tropical Medicine and Hygiene 67: 528-532. https: / / doi.org/10.4269/ ajtmh.2002.67.528

Ramírez-Pulido, J., J. Arroyo-Cabrales \& A. CastroCampillo. 2005. Estado actual y relaciones nomenclaturales de los mamíferos terrestres de México. Acta Zoológica Mexicana (n. s.) 21: 21-82.

Redford, K. H. 1986. Dietary specialization and variation in two mammalian myrmecophages (variation in mammalian myrmecophagy). Revista Chilena de Historia Natural 59: 201-208.

Robinson, J. G. \& E. L. Bennett. 2000. Carrying capacity limits to sustainable hunting in tropical forests. Pp. 13-30 in: Hunting for sustainability in tropical forests (J. G. Robinson \& E. L. Bennett, eds.). Columbia University Press, New York.

Robinson, J. G. \& R. E. Bodmer. 1999. Towards wildlife management in tropical forests. Journal of Wildlife Management 63: 1-13. https://doi. org / $10.2307 / 3802482$

Robinson, J. G. \& K. H. Redford. 1994. Measuring the sustainability of hunting in tropical forests.
Oryx 28: 249-256. https:// doi.org/10.1017/ S0030605300028647

SEMARNAT - Secretaría de Medio Ambiente y Recursos Naturales. 2012. Plan de manejo tipo para armadillo de nueve bandas (Dasypus novemcinctus) modalidad intensiva. 2012. Subsecretaría de gestión para la protección ambiental - Dirección General de Vida Silvestre SEMARNAT, México, D.F. 51 pp.

Staller, E. L., W. E. Palmer, J. P. Carroll, R. P. Thornton \& D. C. Sisson. 2005. Identifying predators at northern bobwhite nests. Journal of Wildlife Management 69: 124-132. http: / / dx. doi.org / 10.2193 / 0022-541X(2005)069<0124:IPANBN $>2.0 . \mathrm{CO} ; 2$

Tanhauser, S. M., M. A. Cheadle, E. T. Massey, B. A. Mayer, D. E. Schroedter, J. B. Dame, E. C. Greiner \& R. J. MacKay. 2001. The nine-banded armadillo (Dasypus novemcinctus) is naturally infected with Sarcocystis neurona. International Journal of Parasitology 31: 325-329. https: / / doi. org/10.1016/S0020-7519(01)00178-3

Tlapaya, L. \& S. Gallina. 2010. Cacería de mamíferos medianos en cafetales del centro de Veracruz, México. Acta Zoológica Mexicana (n. s.) 26: 259-277.

Tyler, J. D., S. Molinaro \& M. Messina. 1996. A note on spring food of armadillos, Dasypus novemcinctus, in a residential area. Proceedings of the Oklahoma Academy of Science 76: 99-101.

Williams-Linera, G., R. H. Manson \& E. Isunza-Vera. 2002. La fragmentación del bosque mesófilo de montaña y patrones de uso del suelo en la región oeste de Xalapa, Veracruz, México. Maderas y Bosques 8: 73-89. https://doi.org/10.21829/ myb.2002.811307

Yeo, M., N. Acosta, M. Llewellyn, H. Sánchez, S. Adamson, G. A. Miles, E. López, N. González, J. S. Patterson, M. W. Gaunt, A. R. de Arias \& M. A. Miles. 2005. Origins of Chagas disease: Didelphis species are natural hosts of Trypanosoma cruzi I and armadillos hosts of Trypanosoma cruzi II, including hybrids. International Journal of Parasitology 35: 225-233. https://doi. org/10.1016/j.ijpara.2004.10.024 